\title{
Zwischen Empirischer Psychologie und Rationaler Seelenlehre. Tetens über das Selbstgefühl
}

Wie sein schottischer Zeitgenosse Thomas Reid betreibt Tetens Philosophie mit historischem Bewusstsein. Immer wieder verortet er seine Position im Kontext der philosophiegeschichtlichen Vorgaben und verweist dabei auf eine Vielzahl von Denkern. In den Beiträgen zu diesem Band ist einiges zu lesen über Tetens und Berkeley, über sein Verhältnis zu Wolff, Reid, Bonnet und anderen. ${ }^{1}$ In der Vorrede zu seinen Philosophischen Versuchen von 1777 bekennt Tetens sich auch zu einer ganz bestimmten Tradition, der des Empirismus. Er spricht sich für eine an der Beobachtung orientierten Herangehensweise aus und stellt sein Hauptwerk als eine "psychologische Analysis der Seele« dar, »die auf Erfahrungen beruhet «. ${ }^{2}$ Des Weiteren sagt er zu seiner Methode:

Was die Methode betrifft, deren ich mich bedient habe, so halte ichs für nöthig, darüber zum voraus mich zu erklären. Sie ist die beobachtende, die Lock bey dem Verstande, und unsere Psychologen in der Erfahrungs-Seelenlehre befolgt haben. Die Modifikationen der Seele so nehmen, wie sie durch das Selbstgefühl erkannt werden; diese sorgfältig wiederholt, und mit Abänderung der Umstände gewahrnehmen, beobachten, ihre Entstehungsart und die Wirkungsgesetze der Kräfte, die sie hervorbringen, bemerken; alsdenn die Beobachtungen vergleichen, auflösen und daraus die einfachsten Vermögen und Wirkungsarten und deren Beziehungen aufeinander aufsuchen. ${ }^{3}$

Dies, so sagt Tetens, seien die »wesentlichsten Verrichtungen « ${ }^{4}$ einer psychologischen Analyse der Seele. Und in der Tat beruft sich Tetens in seinen Versuchen wiederholt auf Erfahrung und Beobachtung. Dies bedeutet allerdings nicht, dass er eine bloße Ansammlung beobachtbarer Fakten anstrebt oder eine bloße Beschreibung der »Wirkungen der Seele« und ihrer »Verbindungen«. ${ }^{5}$ Vielmehr geht es ihm darum, auf dieser Grundlage »Grundsätze zu finden, woraus

1 Vgl. Zum Einfluss der schottischen Common Sense Philosophie auf Tetens vgl. Manfred Kuehn: Scottish Common Sense in Germany, 1768-1800. A Contribution to the History of Critical Pbilosophy. Kingston, Montreal 1987, S. 119-140. Kuehn behandelt nicht den Themenkomplex sSelbstbewusstsein: siehe aber auch den Beitrag von Nele Schneidereit in diesem Band.

2 Johann Nikolaus Tetens: Philosophische Versuche über die menschliche Natur und ibre Entwickelung. 2 Bde. Leipzig 1777, [im Folgenden zitiert als: PV Band, Seitenzahl (Versuch, Kapitel, Abschnitt)], hier Bd. 1, S. IV (Vorrede).

3 Ebd., S. IIIf. (Vorrede).

4 Ebd., S. IV (Vorrede).

5 Ebd. 
sich mit Zuverlässigkeit auf ihre Ursachen schließen« lasse, um dann »etwas gewisses [...] über die Natur der Seele, als des Subjekts der beobachteten Kraftäußerungen, festsetzen« zu können. ${ }^{6}$ Tetens' Ziel scheint also eine Metaphysik auf empirischer Grundlage zu sein, und zur Erreichung dieses Ziels, so betont er, ist offensichtlich nicht nur Beobachtung erforderlich, sondern auch »Raisonnement«. »Am Ende sind es doch die Reflexionen und Schlüsse, die die simpelen Beobachtungen erst recht brauchbar machen, und ohne die wir beständig nur auf der äußern Fläche der Dinge bleiben müßten «. ${ }^{7}$ Tetens möchte nicht an der Oberfläche bleiben, sondern auf Grundlage von »Reflexionen und Schlüssen« etwas über die »Natur der Seele« ausmachen. Bereits mit diesen Hinweisen unterscheidet sich Tetens trotz aller Vorsicht, die er bei Wesensaussagen über die Seele walten lässt, deutlich von John Locke, als dessen deutscher Repräsentant er ja bezeichnet worden ist. ${ }^{8}$ Denn Locke hatte argumentiert, das Wesen der Seele wie auch das der körperlichen Natur sei für den menschlichen Verstand unerkennbar.?

In dem oben zitierten Passus zur Methode kommt der Begriff des Selbstgefühls vor. Tetens' Aussage macht deutlich, dass dieser Begriff für ihn von zentraler Bedeutung für die »psychologische Analysis der Seele« ist. Seine Ausführungen zum Selbstgefühl sind allerdings komplex und weit verzweigt, und es bedarf einiger Anstrengung, sie zu rekonstruieren und die relevanten Begriffsbestimmungen und -relationen aufzuklären. In diesem Beitrag sollen vor allem folgende Fragen untersucht werden: Was ist der Gegenstand des Selbstgefühls? Was für eine Art von Bezugnahme auf seinen Gegenstand stellt das Selbstgefühl dar? Wie verhält sich das Selbstgefühl zum Bezugnehmen auf das Ich durch das, was Tetens Bewusstsein, Selbstbewusstsein und inneren Sinn nennt? Wie verhält es sich zur Wahrnehmung äußerer Gegenstände, die bei Tetens mit dem Terminus »Apperzeption« assoziiert ist? Schließlich wird in kritischer Absicht die Frage zu stellen sein, ob Tetens in seinen Ausführungen zu diesem Themenkomplex nicht nur über das hinausgeht, was durch Erfahrung und Beobachtung direkt beschreibbar ist, sondern auch über das, was aus »Reflexionen und Schlüssen« mit Bezug auf das in der Erfahrung gegebene Material und in Übereinstimmung mit diesem heraus »raisonnirt« werden kann.

Der heute in der Philosophie eher unübliche Terminus »Selbstgefühl« erfreute sich in den 1770er Jahren in Untersuchungen, die im weitesten Sinne mit der empirischen Psychologie zu tun hatten, großer Beliebtheit. Es handelte sich um eine Art Modewort, das noch relativ neuen Datums war. Es wurde wohl von Johann Bernhard Basedow 1764 in die philosophische Terminologie eingeführt, ${ }^{10}$ obwohl ähnliche Ausdrücke wie etwa »inneres Gefühl natürlich schon früher eine Rolle spielten. ${ }^{11}$ Populär dürfte der Begriff durch Johann Georg Heinrich Feder,

6 Ebd.

$7 \quad$ PV I, S. XXX (Vorrede).

8 Vgl. die Hinweise hierzu bei Manfred Kuehn: Hume and Tetens. In Hume Studies 15 (1989), S. 365-376, hier S. 368.

9 Vgl. John Locke: An Essay Concerning Human Understanding. Ed. by Peter H. Nidditch. Oxford 1975. Zitiert wie üblich nach Buch, Kapitel, Sektion, hier: II.i.4.

10 Johann Bernhard Basedow: Philalethie. Neue Aussichten in die Wabrheiten der Religion und Vernunft bis in die Gränzen der glaubwürdigen Offenbarung. 2 Bde. Altona, Bd. 2, \ 10.

11 Georg Friedrich Meier: Metaphysik. 4 Bde. Halle 1755-1759, hier Bd. 3, S. 10 u. S. 21. Isaak Iselin spricht von der »innerlichen Empfindung«, die er bestimmt als »das Bewusstseyn seiner selbst und der Veränderungen, welche in ihm vorgehen«. (Isaak Iselin: Über die Geschichte der Menschheit. Frankfurt, Leipzig 1764, hier zitiert nach der fünften Auflage, Basel 1786, S. 4). 
insbesondere durch seine vielfach aufgelegte und einflussreiche Logik und Metaphysik von 1769 geworden sein. ${ }^{12}$ Schließlich verfasste ein gewisser Michael Ignaz Schmidt 1772 sogar eine Monographie mit dem (aus heutiger Sicht irreführenden) Titel Die Geschichte des Selbstgefühls. ${ }^{13}$ Allerdings ist nicht immer klar, was genau mit diesem Ausdruck gemeint ist. Jedenfalls wird der Terminus von den vielen Autoren, die ihn gebrauchen, in unterschiedlicher Weise gedeutet. Darauf weist der Göttinger Philosoph Michael Hißmann bereits 1777 hin, was ihn jedoch nicht daran hindert, diesen Ausdruck selbst zu verwenden und mit einer wiederum ihm (und Christoph Meiners) eigenen Bedeutung zu versehen. ${ }^{14}$ Diesen komplexen historischen Verflechtungen und den damit verbundenen systematischen Fragen soll hier nicht nachgegangen werden, zumal dies an anderer Stelle bereits geschehen ist. ${ }^{15}$ Hier wird nur auf einige wenige Aspekte verwiesen, die für Tetens besonders relevant sind.

\section{Selbstgefühl und Innerer Sinn: Zum Gegenstand des Selbstgefühls}

Der Begriff des Selbstgefühls wurde oft mit dem Begriff des inneren Sinns in Verbindung gebracht, also mit dem, was Locke »Reflection« nannte. ${ }^{16}$ Basedow wollte den Ausdruck »innerer Sinn« durch »Selbstgefühl« geradezu ersetzen, weil »innerer Sinn« eine unangemessene Analogie zum »äußeren Sinn« nahelege. Für Feder ist das Selbstgefühl nicht mit dem inneren Sinn identisch, sondern eine bestimmte Art des inneren Sinns: er unterscheidet mit Bezug auf diesen die Gefühle des Wahren, Schönen, Guten und eben das Selbstgefühl. Dieses bestehe in der »Gewahrnehmung«, wie Feder sagt, von den eigenen Zuständen und Eigenschaften sowie von der eigenen Existenz. ${ }^{17}$

Tetens scheint das Selbstgefühl, als Vermögen gedacht, in ähnlicher Weise mit dem inneren Sinn zu verbinden. Das Gefühlsvermögen überhaupt ist für Tetens eines der drei Grundvermögen der menschlichen Seele, denen er seinen zweiten Versuch widmet. Er unterscheidet zwischen Gefühl und Empfinden mittels des Objektbezugs. Während sich das Gefühl »mehr auf den Aktus des Empfindens, als auf den Gegenstand desselben« bezieht, weist das Empfinden »auf einen Gegenstand hin«. ${ }^{18}$ Das Selbstgefühl müsste demnach eine Unterart des Gefühls oder der Empfindungsakte überhaupt sein. Und in der Tat argumentiert Tetens, dass das Selbstgefühl zu den »inneren Empfindungen [...] gehöret«. Es sei »das Gefühl jedweder Art von innern Zuständen und Veränderungen für sich betrachtet, so wie sie für sich in uns vor-

12 Johann Georg Heinrich Feder: Logik und Metaphysik. Göttingen, Gotha 1769, S. 116, 267f. Wie aus dem Beitrag von Michael Sellhoff in diesem Band hervorgeht, war Feder eine wichtige Quelle für Tetens.

13 Michael Ignaz Schmidt: Die Geschichte des Selbstgefühls. Frankfurt, Leipzig [i.e. Würzburg] 1772.

14 Michael Hißmann: Psychologische Versuche, ein Beytrag zur esoterischen Logik. Frankfurt, Leipzig 1777, S. 144.

15 Udo Thiel: Varieties of Inner Sense. Two Pre-Kantian Theories. In: Archiv für Geschichte der Philosophie 79 (1997), S. 58-79.

16 Locke: Essay II.i.4

17 Feder: Logik und Metaphysike (s. Anm. 12), S. 116; 7. Aufl., Göttingen 1790, S. 29.

18 PVI, S. 167f. (2. I.) 
handen sind «. ${ }^{19}$ Ähnlich wie Feder fasst Tetens den inneren Sinn oder die innere Empfindung demnach weiter als das Selbstgefühl: Denn zu den Gegenständen der inneren Empfindung gehören auch »die Verbältnisse und Beziehungen« von Objekten, die »in uns selbst sind «. ${ }^{20}$ Hierzu ist beispielsweise das »Gefühl der Einerleyheit und Verschiedenheit« zu zählen. ${ }^{21}$ Des Weiteren schließen die Gegenstände der inneren Empfindungen »die subjektivischen Verhältnisse und Beziehungen der Gegenstände und der Veränderungen auf unsern jetzigen Zustand « ein. ${ }^{22}$ Hierzu gehört wie bei Feder »das Gefübl des Schönen, des Guten, des Wabren«. ${ }^{23}$ Schließlich fühlen wir auch die Gegenstände und Veränderungen in ihrem »Einfluss auf unsere selbstthätige Kraft, auf dieser ihre Wirksamkeit und auf unsere neuen folgenden Zustände, die davon abhangen. Hierher gehört das Gefühl des Interesse, der Wichtigkeit, der Kraft, des Lebens, der Stärke aufs Herz u.s.f.«. ${ }^{24}$

Mit dieser Bestimmung des Selbstgefühls als einer Art innerer Empfindung oder des inneren Sinnes ist wenigstens ein Gegenstandstyp des Selbstgefühls bezeichnet: innere Zustände der Seele, ihre Tätigkeiten und Wirkungen oder Modifikationen. ${ }^{25}$

\section{Was für eine Art von Bezugnahme auf seinen Gegenstand stellt das Selbstgefühl dar?}

Zur Frage nach der Weise des Bezugnehmens auf die »inneren Zustände und Veränderungen«, um die es sich beim Selbstgefühl handelt, hat Manfred Frank behauptet, Tetens' Äußerungen seien nicht einheitlich und widersprächen einander sogar. ${ }^{26}$ Tetens schwanke hin und her zwischen einem Modell der heute sogenannten second-order-Konzeptionen des Bewusstseins einerseits, »wonach Bewusstsein ein zweiter Akt ist, der einen ersten (unbewussten) Akt allererst bewusst macht«, und einem first-order-Modell, wonach Bewusstsein ein wesentliches und »unmittelbares und immanentes Charakteristikum « der mentalen Akte selbst ist. ${ }^{27}$ Es erweist sich jedoch, dass Tetens weder schwankt noch sich widerspricht, sondern zwei unterschiedliche Weisen der Bezugnahme auf innere Zustände voneinander unterscheidet.

Für die Zuschreibung eines second-order-Modells scheinen Tetens' Kommentare über das »Ich denke« des Descartes und über die Descartes-Kritik von Johann Bernhard Merian zu spre-

\footnotetext{
19 Ebd., S. 190 (2. III. 4).

20 Ebd.

21 Ebd.

22 Ebd.

23 Ebd.

24 Ebd., S. 190f.

25 Vgl. auch die Ausführungen zum inneren Sinn in PV I, S. 29f. (1. IV.). Tetens' Darstellung von Lockes »Reflection« ist jedoch problematisch, zumal er diese nicht wie Locke als inneren Sinn, sondern als »die denkende Kraft der Seele« auffasst (ebd., S. 338 [4. VI. 2.]).

26 Manfred Frank: Selbstgefübl. Frankfurt a.M. 2002, S. 199.

27 Ebd., S. 204 u. S. 199.
} 
chen. ${ }^{28}$ Tetens argumentiert ähnlich wie schon Leibniz in seiner Kritik an Locke und Hobbes gegen Descartes, ${ }^{29}$ dass man zu dem Zeitpunkt, an dem man sich eines Gegenstandes bewusst sei (und damit das Denkvermögen auf ihn anwende), nicht denken könne, dass man denke:

Man ist sich nicht bewußt, daß man sich einer Sache bewußt sey; jenes nemlich nicht in demselbigen Augenblick, worinn man dieses ist. Ueber unsere eigene Reflexion reflektiren wir nicht in demselbigen Augenblick, in dem wir mit ihr bey einem Gegenstand beschäftiget sind. ${ }^{30}$

Ein unmittelbares Bezugnehmen auf mentale Zustände oder Akte ist nicht möglich, scheint Tetens zu sagen, gerade weil es sich dabei um eine second-order-Aktivität handeln müsste. Die Seele könne aber nicht einen Gegenstand denken und unmittelbar dieses Denken des Gegenstandes denken, da die Denkkraft dann zwei Tätigkeiten gleichzeitig verrichten müsste. ${ }^{31} \mathrm{Im}$ Sinne einer second-order-Aktivität könne sich Bewusstsein oder Reflexion nur auf vergangene Gedanken beziehen:

Sobald wir auf das Denken selbst zurücksehen, so ist der Gedanke entwischet, wie das gegenwärtige Zeitmoment, das schon vergangen ist, wenn man es ergreifen will. [...] Der Zeitpunkt der Handlung schließet die Reflexion über dieselbige Handlung aus. Diese letztere folget erst auf jene. Hr. Merian hat hierauf seine Kritik über des Descartes Grundsatz: ich denke gebauet, an dessen Statt es seiner Meinung nach heißen müßte: ich habe gedacht. 32

Allerdings spricht Tetens an dieser Stelle gar nicht vom Selbstgefühl, sondern vom Bewusstsein, das er hier als Reflexion und damit als second-order-Aktivität auffasst. Dies lässt (wie bei Merian) die Möglichkeit einer anderen, unmittelbaren Art von Bezugnahme auf innere Zustände offen. Tetens nennt als Beispiel hierfür mentale Zustände wie Lust, Zufriedenheit, Ärger, Liebe usw. Diese fühlen wir, während wir sie haben, ${ }^{33}$ denn sich zu ärgern ist nichts anderes als zu fühlen, dass man sich ärgert. Dementsprechend sagt Tetens: »Die leidenden Gemüthszustände stehen also in einer anderen Beziehung auf das Bewußtseyn, als die Selbstthätigkeiten «. ${ }^{34}$ Aber auch Denkakte sind uns nach Tetens in einem unmittelbaren Sinne präsent, nämlich dann, wenn ich das »Ich denke« nicht als Reflexionsakt auffasse, also damit nicht sagen will, dass ich mein Denken denke oder dass ich weiß, dass ich denke.

Vgl. Johann Bernhard Merian: Mémoire sur l'apperception de sa propre existence. In Histoire de l'Académie Royale des Sciences et Belles Lettres. Année 1749. Berlin 1751, S. 416-441. Zur Merians Apperzeptionsbegriff vgl. Udo Thiel: Between Wolff and Kant: Merian's Theory of Apperception. In Journal of the History of Philosopby 34 (1996), S. 213-232; ders.: The Early Modern Subject. Self-consciousness and Personal Identity from Descartes to Hume. Oxford 2011, S. 365-376.

29 Vgl. hierzu ebd. S. 48 u. S. 299.

30 PV I, S. 46 (1. VII.). Tetens fügt in einer Fußnote auf derselben Seite hinzu: »Man sehe des Hrn Merians Abhandlung darüber, in den Schriften der Berlinischen Akademie der Wissenschaften. 1762«. Vgl. auch PVI, S. 386, wo Tetens ähnlich argumentiert, ohne sich allerdings auf Merian zu beziehen. Tetens' Datierung von Merians Abhandlung ist fehlerhaft. Diese erschien zuerst 1751 auf Französisch (siehe oben, Anm. 28), dann 1778 auf Deutsch als Ueber die Apperzeption seiner eignen Existenz: In Magazin für die Philosophie und ibre Gescbicbte. Aus den Jabrbüchern der Akademien angelegt. Hg. v. Michael Hissmann. Göttingen, Lemgo 1778, Bd. I , S. 89-132.

31 Vgl. Frank: Selbstgefühl (s. Anm. 26), S. 203f.

$32 \quad$ PV I, S. 47 (1. VII.).

33 Ebd., S. 53 (1. VII.).

34 Ebd. 
Ich kann auch in der gegenwärtigen Zeit sagen: ich denke; denn dieß soll nur den Aktus des gegenwärtigen Denkens ausdrücken; nicht aber so viel heißen, als: ich denke, daß ich denke, oder ich weiß, daß ich den$k e .35$

Diese unmittelbare Präsenz ist, wie wir noch sehen werden, bei Tetens mit dem Selbstgefühl gemeint. Damit unterscheidet sich dieses nicht nur von der Reflexion im Sinne eines second-orderAktes des Denkens, sondern auch von dem Lockeschen Verständnis von Reflection als innerem Sinn. Denn auch die Tätigkeit des inneren Sinnes ist bei Locke als second-order-Aktivität aufzufassen. Die Reflexion, dies scheint Tetens zu meinen, setzt aber das Selbstgefühl voraus. Denn dieses gibt erst das Material an die Hand, das dann zum Gegenstand der Reflexion werden kann. Ein Schwanken zwischen den beiden Modellen des Bezugnehmens auf »innere Zustände und Veränderungen« oder gar eine Widersprüchlichkeit liegt bei Tetens jedenfalls nicht vor.

\section{Selbstgefühl im Verhältnis zu Bewusstsein, Apperzeption, Selbstbewusstsein und Reflexion}

Was ist nun bei Tetens mit »Bewusstsein« gemeint? Der Begriff des Bewusstseins schien ja bereits eben in der Descartes-Kritik auf. Wie verhält sich dieser Begriff zu dem des Selbstgefühls? Und wie steht es um die Begriffe der Apperzeption und des Selbstbewussteins und der Reflexion, derer sich Tetens auch bedient?

In Bezug auf Apperzeption und Bewusstsein schließt sich Tetens nun nicht der empiristischen Tradition an, sondern folgt weitgehend Christian Wolff, auch wenn er sich nicht explizit auf Wolff beruft und seine Terminologie gegenüber der Wolffs etwas verrutscht. ${ }^{36}$ Beginnen wir mit der Apperzeption. Diese wird von Tetens nicht, wie es häufig geschieht, als Selbstbewusstsein aufgefasst, sondern als »Gewahrnehmen« (von Gegenständen) übersetzt. In diesem Sinne ist für die Apperzeption (wie bei Wolff für das Bewusstsein) die Aktivität des Unterscheidens wesentlich: »Gewabrnehmen ist ein Unterscheiden«. ${ }^{37}$ Wir apperzipieren einen Gegenstand oder nehmen diesen gewahr, wenn wir seiner Unterschiedenheit von anderen Gegenständen gewahr werden.

Wenn die Seele [...] einen Gegenstand nun als einen besondern Gegenstand fasset, ihn auskennet unter andern, ihn unterscheidet; dann ist dasjenige vorhanden, was ein Gewabrwerden oder ein Gewabrnehmen, oder die Apperception genennet wird. ${ }^{38}$

Da nun das Unterscheiden eine Aktivität der Denkkraft ist, schreibt Tetens das Gewahrnehmen bzw. die Apperzeption der Denkkraft zu, denn das Wesen des Denkens bestehe darin, »Verhältnisse und Beziehungen in den Dingen überhaupt« zu erkennen. ${ }^{39}$ Des Weiteren argumen-

Ebd., S. 48 (1. VII.).

Vgl. zu letzterem beispielsweise schon Falk Wunderlich: Kant und die Bewußtseinstheorien des 18. Jabrbunderts. Berlin, New York 2005, S. 75f. Vgl. zu Wolff auch die Hinweise unten in Anmerkung 43.

PV I, S. 262 (3. I.).

Ebd. Tetens fügt hinzu: »Ohne Zweifel hat dieß Wort [...] ursprünglich eine viel eingeschränktere Bedeutung."

PV I, S. 295 (4. I.). 
tiert Tetens, ähnlich wie Wolff (in Bezug auf das Bewusstsein), dass Aufmerksamkeit und Reflexion notwendige Bedingungen der Apperzeption seien ${ }^{40}$ und dass Reflexion als ein Vergleichen von Dingen oder Vorstellungen von Dingen zu verstehen sei. ${ }^{41}$

Die Apperzeption scheint demnach für Tetens nicht wesentlich einen Selbstbezug zu involvieren, es ist auch gar kein Gefühl, sondern ein intellektuelles Vermögen. Dies verhält sich anders beim Bewusstsein. Tetens bestimmt dieses, anders als der Passus über das »Ich denke« es nahelegt, nicht als Reflexion. Es führt vielmehr zurück zum Selbstgefühl. Das Bewusstsein involviert zwar eine Handlung des Unterscheidens, und damit das Gewahrnehmen oder die Apperzeption. Das Bewusstsein ist aber für Tetens ein mentaler Zustand, in dem man durch die Unterscheidungshandlung nicht nur auf einen Gegenstand oder eine Vorstellung davon Bezug nimmt, sondern auch auf sein eigenes Ich als Subjekt dieser Handlungen. Das Bewusstsein ist ein Gefühl, das das Fühlen des Gegenstandes und des Ich vereine. Es involviere eine Handlung des Unterscheidens zwischen dem gefühlten Gegenstand und dem eigenen Ich:

Sich einer Sache bewußt seyn, drucket einen fortdaurenden Zustand aus, in welchem man einen Gegenstand oder dessen Vorstellung unterscheidend fühlet, und sich selbst dazu. Das Bewußtseyn ist von Einer Seite ein Gefühl, [...] mit dem ein Unterscheiden der gefühlten Sache und Seiner selbst verbunden ist. Gefühl und Gewahrnehmung [also Apperzeption] sind die beiden Bestandtheile des Bewußtseyns. 42

Schon dieses Zitat weist darauf hin, dass für Tetens Gegenstandsbezug und Ich-Bezug »unzertrennlich«, wie er sagt, zusammengehören. Tetens argumentiert, dass es ohne Objekt-Bezugnahme keine Bezugnahme auf das Ich geben könne:

Konnte der Mensch sein Ich kennen, und unterscheiden lernen, ohne zugleich einen Begrif von einem wirklichen Objekt zu erhalten, das nicbt sein Ich ist? Und wenn diese beiden Begriffe unzertrennlich sind, so war es doch eben so möglich, daß die beiderley Arten von Urtheilen; dieß ist in mir, und: jenes ist nicht in mir, zu gleicher Zeit sich entwickelt hatten, ohne daß das letztere das erste voraussetze, und nachher mittels anderer Gedanken, die noch gesammelt werden mußten, hervorgebracht werden dörfe. ${ }^{43}$

Das Selbstgefühl ist mit dem Selbstbewusstsein jedoch nicht einfach gleichzusetzen. Hierzu schreibt Tetens:

40 Ebd., S. 282-285 (3. IV. 2.).

41 Ebd., S. 264 (3. II.). Tetens unterscheidet zwischen Gewahrnehmen und Bemerken. »Das Bemerken will etwas mehr sagen, als Gewahrnehmen. Wer etwas bemerket, suchet an der gewahrgenommenen Sache ein Merkmal auf, woran sie auch in der Folge gewahrgenommen und ausgekannt werden könne« (ebd., S. 263 [3. I.]).

42 Ebd.

43 Ebd. S. 379 (5. II.). Für ein ähnliches Verhältnis von Objekt- und Ich-Bezug hatte bereits Wolff argumentiert, allerdings sagt er nicht nur, dass die beiden Begriffe unzertrennlich sind und dass wir keinen Ich-Bezug ohne Objekt-Bezug haben, sondern auch, dass wir ohne Ich-Bezug keinen ObjektBezug haben können. Er legt also eine wechselseitiges Abhängigkeitsverhältnis nahe, ohne dies freilich im einzelnen zu erläutern; vgl. Christian Wolff: Vermünfftige Gedancken von Gott, der Welt und der Seele des Menschen, auch allen Dingen überhaupt. Halle 1751 [ND Hildesheim 1983], \ 730. Vgl. dazu Udo Thiel: Zum Verbältnis von Gegenstandsbewußtsein und Selbstbewußtsein bei Wolff und seinen Kritikern. In: Jürgen Stolzenberg u. Oliver-Pierre Rudolph (Hg.): Christian Wolff und die Europä̈sche Aufklärung. Akten des 1. Internationalen Christian-Wolff-Kongresses. 5 Tle. Hildesheim, New York 2007-2010, Tl. 2, S. 377-390; Thiel: The Early Modern Subject (s. Anm. 28), S. 304-311; Wunderlich: Kant und die Bewnußseinstheorien (s. Anm. 36), S. 77. 
Es sind alsdenn klare Empfindungen und klare Empfindungsideen, Wirkungen aus Perception, Gefühl und Apperception zusammengesetzt, so wie das vorzüglich starke Gefühl unserer Selbst nicht mehr ein bloßes Gefühl, sondern ein klares Gefühl, eine Empfindung, ein Bewußtseyn unsers Selbst ist. Denn es vereiniget sich mit dem Gefühl das Unterscheiden der gefühlten Modifikation und des fühlenden Subjekts, und die Beziehung jener Modifikation auf das Subjekt, worinn sie ist. ${ }^{44}$

Das Bewusstsein enthält für Tetens neben dem Gegenstandsbezug bereits ein unmittelbares Selbstgefühl. Im Selbstbewusstsein werden noch einmal die Modifikationen des Subjekts vom fühlenden Subjekt unterschieden und auf es bezogen. Erst durch diese Bezugnahme wird aus dem Selbstgefühl ein Selbstbewusstsein. Gegenüber dem (unmittelbaren) Selbstgefühl ist das Selbstbewusstsein bei Tetens eine vermittelte Bezugnahme auf das Ich. ${ }^{45}$

Schließlich unterscheidet Tetens zwischen dem starken und unmittelbaren Selbstgefühl einerseits und dem Reflektieren auf das Ich, das uns befähigt, Aussagen über uns formulieren zu können andererseits. Dies entspricht der oben diskutierten Unterscheidung, wonach Bewusstsein als Denken des Denkens und damit als Reflexion und second-order-Aktivität gilt, während das Selbstgefühl unmittelbar ist. Die Reflexion verdankt sich dem Denkvermögen oder der »Denkkraft«. Der Gedanke, dass dies ich bin und dass diese Gedanken in mir vorgehen, setze sorgfältige Selbstreflexion voraus. Die Erfahrung zeige,

daß die Reflexion der Seele über sich selbst und insbesondere der Gedanke, das bin ich, und das ist in mir, und geht in mir vor, ebenfalls zu den Wirkungen der Denkkraft gehöre, wozu diese sich nur nach und nach entwickelt, und daß eine sorgfältige Beobachtung seiner selbst voraussetze, daß man schon aus der Kindheit heraus sey. Aber wer wird darum das starke Selbstgefühl in Zweifel ziehen?46

\section{Das Ich als Gegenstand des Selbstgefühls. Tetens' Kritik an Humes Bündeltheorie.}

Die obigen Ausführungen über das Bewusstsein machen es erforderlich, dass wir zur Frage nach dem Gegenstand des Selbstgefühls zurückkehren. Denn bislang wurden ja nur innere Zustände und Tätigkeiten als sein Gegenstand dargestellt (»die innern Modifikationen, deren Gefühl unser Selbstgefühl ausmachet«). ${ }^{47} \mathrm{Im}$ Bewusstsein soll aber laut Tetens ein Fühlen des Ich als des Subjekts dieser Zustände und Tätigkeiten enthalten sein. Im Rahmen seiner Kritik an Humes Bündel-Theorie führt Tetens diesen Gedanken weiter aus.

Es verhält sich nicht so, wie es Hr. Hume angegeben hat, und dieß kann man behaupten, ohne etwas mehr für wirklich vorhanden anzunehmen, als was er selbst dafür erkennet; nur so viel nemlich, als wir uns unmittelbar bewußt sind. Hr. Hume hat aber einen wichtigen Umstand übersehen. Ich fühle eine Vorstellung; noch eine andere, auch eine Denkungsthätigkeit, eine Willensäußerung, u.s.w. und diese Empfindungen sind unterschieden, und wirklich. Aber ich empfinde noch mehr. So oft ich eine Vorstellung empfinde, gewahrnehme, und mich ihrer unmittelbar bewußt bin, so bin ich mir eben so gut bewußt, daß dieß Gefühl meiner Modifikation nur ein hervorstechender Zug in einem viel größern,

PV I, S. 298f. (4. II.).

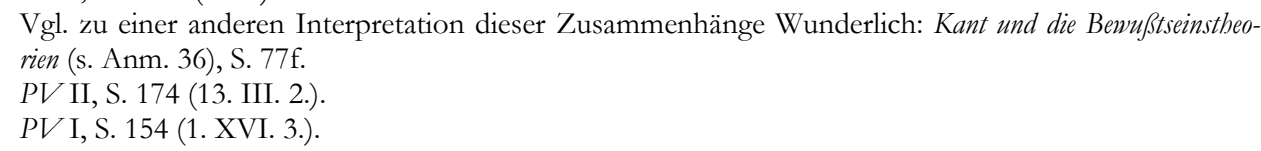


ausgebreitetern, stärkern, obgleich in seinen übrigen Theilen dunklen, oder doch wenig klaren Gefühl sey; und dieses letztere bin ich mir eben so bewußt, und auf dieselbige Art, wie ich es in Hinsicht der besonders gewahrgenommenen einzelnen Beschaffenheit nur immer seyn kann, so nemlich wie man sich überhaupt einer Sache unmittelbar bewußt seyn kann. Ich habe also eine solche Empfindung, die mich auf die nemliche Art zu dem Gedanken bringet, daß ein Ding und eine Beschaffenheit in diesem Dinge vorhanden ist, als ich nach Hrn. Hume's eigener Einräumung zu dem Gedanken gebracht werden kann: da ist eine Beschaffenheit wirklich.

Und in dieser ganzen Empfindung ist der dunkle Grund von ihr immer eben derselbige, wenn ich anstatt eines sich ausnehmenden Zuges einen andern verschiedenen in mir als gegenwärtig gewahrnehme. Dieser Grund der ganzen Empfindung [...] ist bey allen besondern Veränderungen, in der Empfindung und in der Vorstellung eben derselbige. 48

Tetens scheint hier gegen Hume zu argumentieren, dass das Selbstgefühl zum Begriff eines identischen Ich führe und dass Hume diese Tatsache einfach »übersehen« habe. Man könne dies zeigen, ohne dass man mehr an Erfahrungsgegebenheiten akzeptieren müsse als Hume, mit anderen Worten, auch dann, wenn wir uns nur auf die Dinge bezögen, derer wir uns unmittelbar bewusst sind. Wir fühlten, wie Hume ja zugestehen würde, eine Vielheit von Perzeptionen, doch fühlten wir mehr als nur diese. Wenn immer wir eine Perzeption fühlten, seien wir uns auch dessen bewusst, dass dieses Gefühl Teil eines »größeren« und »stärkeren«, wenn auch »dunkleren« Gefühls von einem Grund unserer Perzeptionen sei, der bei allen Veränderungen der Perzeptionen derselbe bleibe. ${ }^{49}$ Daher sei die Vorstellung von unserem Ich mehr als eine bloße Ansammlung von Perzeptionen, die die Einbildungskraft zusammengefügt habe:

Eine $[. .$.$] Folge davon ist, daß die Idee oder Vorstellung von meinem Ich, keine Sammlung von einzeln$ Vorstellungen sey, welche etwan die Einbildungskraft zu einem Ganzen gemacht hat, wie sie die einzelnen Vorstellungen von Soldaten zu einer Vorstellung von Einem Regiment vereiniget. Jene Vereinigung liegt in der Empfindung selbst, in der Natur, nicht in einer selbst gemachten Verbindung. Daher entstehet eine Vorstellung von Einem Subjekt mit verschiedenen Beschaffenheiten, das heißt, die aus der Empfindung unmittelbar entstehende Vorstellung muß so gedacbt, und zu einer solchen Idee gemacht werden, wozu der gemeine Menschenverstand sie wirklich machet. ${ }^{50}$

Tetens stimmt Hume demnach zu, dass von dem auszugehen sei, was unmittelbar dem Bewusstsein gegeben ist. Er kritisiert allerdings Humes Auffassung, dass nur Ansammlungen von einzelnen Perzeptionen gegeben seien. ${ }^{51}$ Ohne sich auf Hume zu beziehen, behauptet Tetens an

48 PV I, S. 393f. (5. V.). Vgl. zum Gedanken des »dunklen Grundes« auch Alexander G. Baumgarten, der in seiner Metaphysica (1. Aufl. 1739) die Gesamtheit der dunklen Perzeptionen den »Grund der Seele« (fundus animae) nennt. Alexander Gottlieb Baumgarten: Metaphysica. Historisch-kritische Ausgabe. Übers., eingel. u. hg. von Günter Gawlick u. Lothar Kreimendahl. Stuttgart-Bad Cannstatt 2011, S. 270 (\$ 511). Vgl. zu diesem Thema Frank: Selbstgefübl (s. Anm. 26), S. 203 f.

49 Vgl. auch PV I, S. 261 (2. VIII.): »Begleitet nicht ein gewisses dunkles Selbstgefühl alle unsere Zustände, Beschaffenheiten und Veränderungen von der leidentlichen Gattung? «

50 Ebd, S. 394 (5. V.).

51 In diesem Punkt stehen wir Manfred Kuehns Interpretation näher als der Falk Wunderlichs. Kuehn argumentiert, dass »Tetens does not so much offer a fundamental criticism of Hume's approach or philosophical method, as claim that Hume has made a mistake in one of the particulars (Kuehn: Hume and Tetens [s. Anm. 8], S. 367). Wunderlich meint, diese Lesart sei »untertrieben«, da die Annahme, dass die Vorstellungen »vereinzelt« auftreten, »eine zentrale Prämisse Humes« sei (Wunderlich: Kant und die Bewußtseinstheorien [s. Anm. 36], S. 77). Es geht Hume jedoch wie Tetens um das, was uns unmittelbar präsent ist. Dass nur aufeinanderfolgende (nicht »vereinzelte«) Vorstellungen gegeben sind, ist für Hume eine Tatsache des Bewusstseins, aber keine »Prämisse«. 
anderer Stelle, dass das Ich als leib-seelische Ganzheit den Gegenstand des Selbstbezugs ausmacht (und nicht nur aufeinanderfolgende Vorstellungen): »Es ist $\operatorname{der}$ Mensch, der von $\operatorname{dem}$ Menschen gefühlet wird «. ${ }^{2}$ Oder, etwas genauer formuliert:

Wenn ich mich selbst und meine Aktionen fühle, was ist alsdenn das Objekt meines Gefühls? Die reine Beobachtung kann [...] nichts anders antworten, als es sey das Ich, was ich fühle, das fühlende, denkende und wollende Ganze, das aus einem Körper und aus einer einfachen Seele bestehet, die eingekörperte Seele. 53

Im 18. Jahrhundert war die These, dass eine angeblich einfache seelische Substanz Gegenstand eines unmittelbaren Gefühls sei, durchaus verbreitet. Sie stellt jedoch offensichtlich eine recht problematische Behauptung dar. Tetens drückt sich gelegentlich etwas vorsichtiger aus und versucht, soweit es um das Selbstgefühl geht, metaphysisch neutral zu bleiben:

Die menschliche Seele im psychologischen Verstande genommen [i.e. die Seelennatur des Menschen], ist das $I c h$, das wir mit unserm Selbstgefühl empfinden und beobachten können. Es mag aus einem einfachen immateriellen Wesen allein bestehen, oder aus diesem, und einem innern körperlichen Werkzeug des Gefühls und des Denkens zusammengesetzt seyn, oder, um kein psychologisches System auszuschließen, es mag nichts als der innere organisirte Körper seyn. Genug es ist das fühlende, denkende und wollende Eins, der innere Mensch selbst. ${ }^{54}$

Hiernach ist zu unterscheiden zwischen dem, was dem Selbstgefühl zugänglich ist, und dem, was dem Gefühlten ontologisch zu Grunde liegt. Die reale Essenz, wie Locke gesagt hätte, mag so oder so beschaffen sein, dies ist letztlich nicht relevant für das Selbstgefühl. Tetens selbst unterscheidet von der Seele als Gegenstand des Selbstgefühls, also der Seele im psychologischen Verstande, die »Seele im metaphysischen Verstande«, 55 die nicht Gegenstand des Selbstgefühls sei. Bei der Seele im psychologischen Verstande handelt es sich nur um »Erscheinungen«:

Was endlich die Natur unsers Selbstgefühls und der Vorstellungen betrifft, die wir von unsern eigenen Wirkungen haben, so können sie [...] nichts mehr als Schein seyn; so wie die unmittelbare Beobachtung uns auch nicht berechtiget, sie für etwas mehr anzusehen [...]. Denn wir empfinden die Aktus unsers Gefühls, und des Denkens, und des Wollens nur in ihren Wirkungen, das ist, in den Veränderungen und Folgen, die davon in dem gesammten Seelenwesen, das ist, in einem zusammengesetzten Wesen abhangen. ${ }^{56}$

Diese »Erscheinungen« beziehen sich zwar »mittelbar auf die Beschaffenheiten, Kräfte und Vermögen des einfachen Ichs«, und insoweit sind sie Vorstellungen von dem Einfachen, aber nur in einem indirekten oder relativen Sinne. ${ }^{57}$ Dennoch nimmt die Behauptung dieser mittelbaren Beziehung offensichtlich die Existenz eines einfachen Ich an. Und in der Tat läuft Tetens' Denken letztlich auf einen Seelenbegriff im metaphysischen Verstande hinaus. Hiernach ist die Seele das »einfache, von dem organisirten Körper unterschiedene [also immaterielle] Wesen «. ${ }^{58}$

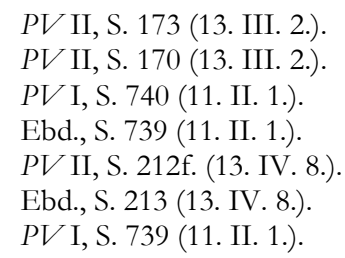


Diese Überlegungen beziehen sich auch auf die von Tetens schon in seiner Hume-Kritik behauptete Einheit des Ich.

\section{Ist die Einheit des Ich als Gegenstand des Selbstgefühls oder als notwendige Bedingung mentaler Aktivitäten zu denken?}

Wie kommen wir nun im Denken von der Seele im psychologischen Verstande, der »beobachteten Einheit des Ichs«, zur Seele im metaphysischen Verstande, zur »substantiellen Einheit der Seele«?59 Zum einen scheint das unkörperliche Ich insofern Gegenstand des Selbstgefühls zu sein, als es Teil des Ganzen ist, das der Mensch fühlt, wenn er den Menschen fühlt, wie wir oben sahen. An anderer Stelle argumentiert Tetens jedoch, dass das unmittelbare Selbstgefühl auf ein unkörperliches Ich nur verweise, dass der Gedanke eines solchen Ich sich »aufdränge«:

Sobald das Gefühl von Ich, das klare Bewußtseyn unser selbst, unsers innern Wohls und Wehs, unsers Denkens und Wollens und unserer Freyheit wieder lebhaft wird, so dränget sich uns auch wiederum der Gedanke auf: dieß sey doch mehr als ein Spiel der Fasern, mehr als ein Zittern vom Aether und als Gehirnsbewegungen, was dahinter stecke. Mein Ich ist Eins, nicht ein Haufen von mehrern Dingen. ${ }^{60}$

Hiernach legt das Selbstgefühl den Gedanken von einem »Eins« wenigstens sehr nahe; aber schon dies bedeutet, dass die unkörperliche Einheit jedenfalls nicht selbst Gegenstand des Selbstgefühls ist. Zum dritten scheint für Tetens gar »theoretische Spekulation über die Natur des Seelenwesens« erforderlich zu sein, um zum Gedanken der substantiellen Einheit des Ich zu kommen. ${ }^{61}$ Tetens versucht, den Einheitsgedanken (und andere metaphysische Thesen) durch rationale Argumentation zu erweisen, die auf die Bedingungen nicht speziell der Erfahrung, sondern des Denkens überhaupt zielt. Er fragt sich, wie aus der beobachteten auf die substantielle Einheit »gefolgert werden könne«. ${ }^{62}$ Wenn auf die Einheit der Seele »gefolgert« werden muss, ist sie offensichtlich nicht unmittelbar im Selbstgefühl gegeben. Tetens' Argumentation ist hier zwar nicht besonders originell, geht aber über seine anfangs deklarierte empiristische Methode hinaus. Zunächst geht es nur darum zu zeigen, dass das Vorstellen äußerer Objekte ein Urteilen voraussetze und dass es hierfür wiederum einer Verknüpfungs- und Unterscheidungsleistung zwischen Ding außer mir, Sache in mir und Selbst bedürfe.

Mit allen Vorstellungen des Gesichts, des Gefühls und der übrigen Sinne wird der Gedanke verbunden, daß sie äußere Objekte vorstellen. Dieser Gedanke bestehet in einem Urtheil, und setzet voraus, daß schon eine allgemeine Vorstellung von einem Dinge, von einem wirklichen Dinge, und von einem äußern Dinge, vorhanden, und daß diese von einer andern allgemeinen Vorstellung von unserm Selbst, und von einer Sache in uns, unterschieden sey. ${ }^{63}$

Hier geht es zwar noch nicht um die »substantielle Einheit der Seele«, aber doch um einen Begriff vom »Selbst«, das nicht als Gegenstand eines Gefühls angesehen wird, sondern als etwas,

$59 \quad$ PV II, S. 175 (13. IV.).

60 Ebd., S. 178 (13. IV. 1.).

61 PV I, S. 740 (11. II. 1.).

62 Ebd.

63 Ebd., S. 344 (4. VI. 3.). 
das zur Erklärung des bloßen Vorstellens äußerer Objekte gedacht werden muss. Ohne einen solchen Begriff vom Selbst, als unterschieden von der Vorstellung anderer Dinge gefasst, ließe sich die Möglichkeit des Urteilens über die Existenz äußerer Gegenstände nicht denken.

Tetens versucht aber darüber hinaus noch zu zeigen, dass auch die substantielle Einheit der Seele gedacht werden müsse. Denn ohne diese wären die Handlungen der Seele nicht möglich: „Die kollektiven Kräfte und Wirkungen setzen eine substantielle Einheit voraus, in der die Kollektion geschieht und in Hinsicht auf welche sie nur solche Kräfte und Wirkungen sind, als sie sind «.64 Selbst für Einzelhandlungen ist eine solche Einheit vorausgesetzt. Wenn Handlungen des Geistes »aus einer Menge anderer Kraftäußerungen zusammengesetzt« sind, können diese Bestandteile nur dann zu einem Akt werden, wenn sie einer substantielle Einheit angehören:

Denn wenn die verschiedenen Bestandtheile des Aktus durch mehrere verschiedene Wesen vertheilet sind, davon jedes einzeln, nur einen einzelnen von jenen Aktus hervorbringet: so ist zwar ein Haufen von Elementen des Gefühls in mehrern Dingen vertheilte vorhanden; aber nirgends ist ein Gefühl, nirgends das vereinigte Ganze aus ihnen, das nach der Voraussetzung, heterogen von seinen Elementen, erst ein Gefühl wird, wenn jene Elemente zusammen genommen werden. ${ }^{65}$

Diese Argumentation ist wie angedeutet keineswegs originell. Sie findet sich in ähnlicher Form bei verschiedenen anti-materialistisch orientierten Denkern des 18. Jahrhunderts. Kants Lehrer Martin Knutzen beispielsweise argumentiert lange vor Tetens, dass die Unterscheidungshandlung (und damit das Denken überhaupt) die »absolute Einheit des Subjekts« voraussetze. Einmal in dem Sinne, dass die Vorstellungen der Dinge in einem einheitlichen Subjekt existieren müssten; denn ohne eine solche Einheit der Vorstellungen wäre ein Unterscheiden zwischen einzelnen Vorstellungen gar nicht möglich; und zweitens insofern es eines aktiven Subjekts bedürfe, das sowohl die Handlungen des Unterscheidens und Vergleichens ausübt als auch die mentalen Akte, die auf diese Weise unterschieden und verglichen werden. Ähnlich wie später Tetens versucht Knutzen zu zeigen, dass diese Einheit des Subjekts nichts anderes als die Einheit einer immateriellen Seelensubstanz sei. ${ }^{66}$

Der Gedanke, dass ein einheitliches und identisches Ich in jeder Urteilshandlung als Bedingung vorausgesetzt ist, wird bekanntlich von Kant in einem anderen systematischen Kontext weiterentwickelt, ohne dass auf die Substantialität oder gar Immaterialität der Seele geschlossen wird. Kant war, wie wir wissen, mit Knutzen und Tetens und anderen Denkern, die ähnlich argumentierten (Rousseau), vertraut. Es ist daher müßig, einen von diesen als seinen besonderen diesbezüglichen »Vorläufer« identifizieren zu wollen. Darüber hinaus darf nicht vergessen werden, dass Tetens, Rousseau und Knutzen im Gegensatz zu Kant diese Argumentation im Rahmen eines anti-materialistischen metaphysischen Programms entwickeln.

Im Übrigen ist diese Argumentation für die immaterielle Substantialität der Seele kaum überzeugend. Schon Materialisten des 18. Jahrhundert weisen darauf hin. Joseph Priestley beispielsweise, einer der wichtigsten materialistischen Denker in der zweiten Hälfte des 18. Jahrhunderts, der großen Einfluss auf die deutsche Diskussion ausübte, argumentierte: Selbst wenn wir eine notwendige Einheit des Ich denkend voraussetzten oder sogar ein Einheitsgefühl kon-

Martin Knutzen: Philosophische Abhandlung von der immateriellen Natur der Seele. Königsberg 1744, S\$ 3, 18.

Zu Knutzen vgl. auch Thiel: The Early Modern Subject (s. Anm. 28), S. 329-331. 
zedierten, folge daraus und aus den Aktivitäten der Seele keineswegs deren Einfachheit und Immaterialität. Aus einem solchen Einheitsgefühl könne allenfalls dies geschlossen werden, dass man nur eine Person, ein empfindendes und denkendes Wesen sei und nicht zwei Personen oder zwei empfindende Wesen. Zwar könne in der Tat ein empfindendes Wesen nicht in zwei empfindende Wesen aufgeteilt werden, da eine solche Teilung das eine ssystem of intelligence which we call the soul of man zerstören würde, aber natürlich könne die Seele des Menschen so geteilt oder aufgelöst werden »as to become no system of intelligence at all ${ }^{67}$

\section{Schluss}

Wir sahen, dass es für Tetens erstens eine empirische Einheit des Ich als Tatsache des Bewusstseins gibt: »Es kann als ein unläugbarer Erfahrungssatz angesehen werden, daß unser Ich sich selbst als ein fühlendes und denkendes Wesen erscheine«. ${ }^{68}$ Es zeige »sich unmittelbar aus den Beobachtungen eine gewisse Einheit unsers Ichs«. ${ }^{69}$ Und so überrascht es nicht, dass Tetens folgende Aussage als »Beobachtungssatz« ansieht:

Ich, der ich fühle, denke, afficirt werde, leide, handle, bin so sehr Eins und dasselbige Wesen, Ding oder Kraft, wie man es nennen will, daß ich keinen Begriff von einer größern Identität habe, als diese Identität meines Ichs ist. ${ }^{70}$

Schon dieser Gedanke der Einheit des Ich im psychologischen Verstande wirft allerdings eine Frage auf: ob sie wirklich Gegenstand der bloßen Beobachtung oder des Gefühls sein kann, wie Tetens behauptet. Fühlen wir diese Einheit oder nicht doch bloß die verschiedenen, aufeinander folgenden inneren Modifikationen, wie Hume wohl gegen Tetens argumentiert hätte? Kurz: Handelt es sich dabei mehr um eine behauptete als eine »beobachtete« Einheit?

Des Weiteren ließe sich einwenden: Selbst wenn wir mit Tetens annähmen, es gebe ein Gefühl, das uns bedeute, wir seien kein »Haufen von mehrern Dingen «, ${ }^{71}$ sondern ein einheitliches Wesen, so könnte ein solches Gefühl uns doch täuschen. Wir könnten uns auf seiner Grundlage für einheitlich und identisch halten, ohne es aber tatsächlich zu sein. Tetens hielte dagegen, dass dieses Gefühl nicht bloß illusorisch sein könne, sondern auf eine reale Einheit verweise. Denn die Existenz des Gefühls fiele weg, sagt er, »wenn nichts weiter, als eine Menge von Wesen da ist, deren jedwedes allein für sich ganz etwas anders als ein Fühlen hervorbringt « ${ }^{72}$

Dennoch gesteht Tetens zu, dass der Hinweis auf die »beobachtete Einheit« nicht ausreiche, sondern dass es zweitens einer rationalen Argumentation bedürfe, die zur »substanziellen Einheit« der Seele, zur Seele als immaterieller Substanz führe. ${ }^{73}$ Wie gesehen führt für Tetens die rationale Analyse mentaler Akte zum Gedanken der Notwendigkeit einer solchen substantiellen Ein-

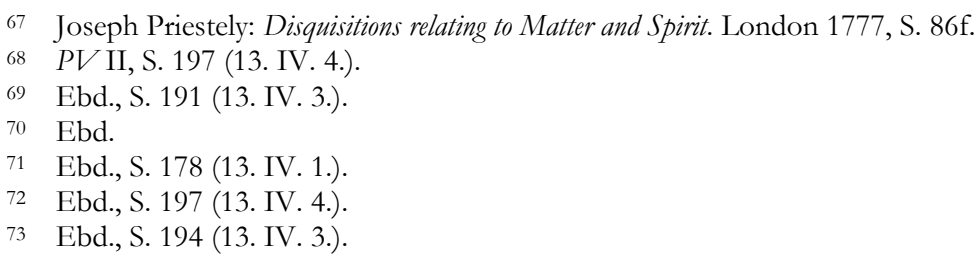


heit. Die Frage, die im Titel des vorherigen Abschnitts gestellt wurde, lässt sich also so beantworten, dass Tetens die Einheit des Ich, in jeweils unterschiedlicher Hinsicht, sowohl als Gegenstand des Selbstgefühls als auch als notwendige Bedingung mentaler Aktivitäten gilt.

Eingangs wurde darauf hingewiesen, dass es Tetens um eine Verbindung von "psychologischer Analyse« und »theoretischer Spekulation« gehe. Diese Hinweise gelten nach dem Gesagten nicht zuletzt für seine Ausführungen zum Selbstgefühl. Tetens kann nur dann als reiner Empirist missverstanden werden, wenn man seine Äußerungen zur Rolle von Erfahrung und Beobachtung isoliert betrachtet. In Ueber die allgemeine speculativische Philosophie betrachtet Tetens die empiristische Herangehensweise nur als einen ersten Schritt in seinen Untersuchungen. Dann komme es auf »die Speculation aus allgemeinen Gründen« an. Hier behauptet Tetens, vielleicht im Anschluss an Leibniz, sogar von den »Scholastikern« könne man hier noch etwas lernen. ${ }^{74}$ Wie man sich das Verhältnis von empiristischer Methode und »Speculation aus allgemeinen Gründen« im Einzelnen vorzustellen hat, wird allerdings nicht ganz klar, auch das gilt für seine Ausführungen zum Selbstgefühl. Klar ist jedoch, dass in Bezug auf den Themenkomplex sSelbstbewusstsein ‘ Tetens' Verhältnis zur Tradition des Empirismus uneindeutiger und komplexer ist, als es zunächst erscheinen mag. Das sollte auch berücksichtigt werden, wenn man das Verhältnis Tetens-Kant betrachtet.

74 Johann Nikolaus Tetens: Ueber die allgemeine speculativische Philosophie. Bützow, Wismar, 1775, S. 86. Vgl. auch S. 73 u. S. 89. Tetens betont: »Die Brittischen Philosophen mögen unsre Muster im Beobachten seyn; aber sie sollten es nicht seyn in der speculativischen Philosophie« (ebd., S. 87). 